PROCEEDINGS OF THE

AMERICAN MATHEMATICAL SOCIETY

Volume 133, Number 10, Pages 3027-3035

S 0002-9939(05)07873-1

Article electronically published on March 31, 2005

\title{
ISOCHRONICITY OF A CLASS OF PIECEWISE CONTINUOUS OSCILLATORS
}

\author{
FRANCESC MAÑOSAS AND PEDRO J. TORRES \\ (Communicated by Carmen C. Chicone)
}

\begin{abstract}
Motivated by a classical pendulum clock model suggested by Andrade in 1920 , we study the equation $\ddot{x}+g(x) \operatorname{sgn} \dot{x}+x=0$ and prove that for a nonlinear analytic $g$ the origin is never an isochronous focus or an isochronous center.
\end{abstract}

\section{INTRODUCTION}

The measurement of time (chronometry) has been an enormous incentive for the development of physics and mathematics. An important advance in this field was the discovery of the fundamental properties of the pendulum by Galileo in 1583 . Later, many other scientists (especially Huygens and Hooke) took advantage of the natural oscillations of different kinds of pendula and springs in their experiments and designs.

From the mathematical point of view, systems with the property that all solutions are periodic with the same period are called isochronous. Isochronous centers, and more generally the study of the so-called period function for potential systems, have received the attention of many researchers 4, 5, 6, 9, 11, 12. However, from the practical point of view, every classical mechanical device in clockmaking is subjected to a loss of energy due to friction. So, it is natural to look for a notion of isochronous dissipative oscillator. In a classical paper [3], Jules Andrade proposed the equation

$$
\ddot{x}+\epsilon|x| \operatorname{sgn} \dot{x}+x=0
$$

as a first such example with practical applications. Looking at the phase plane, the origin is a global attractor and every non-trivial solution completes a whole turn in a fixed time. The dissipation term $\epsilon|x|$ sgn $\dot{x}$ models the so-called dry friction (also

Received by the editors March 1, 2004 and, in revised form, May 27, 2004.

2000 Mathematics Subject Classification. Primary 34C05, 34C15.

Key words and phrases. Isochronous, center, focus.

The first author was partially supported by DGES No. BFM2002-04236-C02-2, BFM200201344 and the CONACIT grant number 2001SGR-00173.

The second author was partially supported by D.G.I. BFM2002-01308, Ministerio Ciencia y Tecnología, Spain. 
known as Coulomb friction; see for instance [8, 10] and the references therein) with a damping coefficient that varies with position. A natural question is to characterize the set of functions $g$ such that

$$
\ddot{x}+g(x) \operatorname{sgn} \dot{x}+x=0
$$

is a dissipative isochronous oscillator, or more generally, a dissipative isochronous focus. As a planar system, the previous equation can be written as

$$
(\dot{x}, \dot{y})= \begin{cases}(-y, x+g(x)) & \text { if } y \geq 0 \\ (-y, x-g(x)) & \text { if } y<0 .\end{cases}
$$

Hence, it is a piecewise smooth planar system whose phase plane is composed of two "uncoupled" smooth systems matched by the line $y=0$. More generally, we will consider a planar system

$$
(\dot{x}, \dot{y})= \begin{cases}\left(f_{+}(x, y), g_{+}(x, y)\right) & \text { if } y \geq 0 \\ \left(f_{-}(x, y), g_{-}(x, y)\right) & \text { if } y<0\end{cases}
$$

such that

(i) $f_{ \pm}, g_{ \pm}$are analytic in some neighborhood of the origin and $f_{ \pm}(0,0)=$ $g_{ \pm}(0,0)=0$, and

(ii) the origin $(0,0)$ is a non-degenerate center (that is, with a non-singular linear part) for the "uncoupled" planar systems $(\dot{x}, \dot{y})=\left(f_{+}(x, y), g_{+}(x, y)\right)$ and $(\dot{x}, \dot{y})=\left(f_{-}(x, y), g_{-}(x, y)\right)$.

By assumption (ii), the system

$$
(\dot{x}, \dot{y})=\left(f_{+}(x, y), g_{+}(x, y)\right)
$$

(respectively, $\left.(\dot{x}, \dot{y})=\left(f_{-}(x, y), g_{-}(x, y)\right)\right)$ has a well-defined period map $T_{+}(x)$ (resp. $T_{-}(x)$ ). If this period map is constant, the center is called isochronous. Let us formalize the notion of isochronous focus.

Definition 1.1. Let $(x(t), y(t))$ denote the solution of system (4) with initial conditions $x(0)=x_{0}>0$ (resp. $\left.x_{0}<0\right)$ and $y(0)=0$. Also, let $T\left(x_{0}\right)$ denote the first return time to the section $\{y=0, x>0\}$ (resp. $\{y=0, x<0\}$ ). System (4) is called an isochronous oscillator if $T\left(x_{0}\right)$ is constant for all $x_{0} \neq 0$ and an isochronous focus if moreover

(iii) the origin $(0,0)$ is a focus for the "coupled system" (4).

System (4) is called an isochronous dissipative oscillator if such a focus is an attractor.

In general, the origin of (4) can be a center, a focus, or the so-called center-focus, where there are infinitely many limit cycles accumulating at the origin. This last possibility does not occur in our setting because of the analyticity of the return map of the coupled system [7].

The main result of this paper is the following theorem.

Theorem 1.1. If $g(x)=\sum_{n \geq 2} a_{n} x^{n}$ is a non-linear analytic function, then system (2) is neither an isochronous focus nor an isochronous center.

This result will be proved using a sequence of partial results distributed in Sections 2 and 3. Section 2 is devoted to the study of isochronous foci whereas Section 3 deals with isochronous centers. 


\section{ISOCHRONOUS FOCI}

In principle, it could happen that system (4) is an isochronous focus but both uncoupled systems are not isochronous. Under some special conditions, our first result states that this is not possible.

Lemma 2.1. Suppose that system (4) satisfies conditions (i), (ii), and (iii). If $f_{ \pm}, g_{ \pm}$are analytic functions such that

$$
f_{ \pm}(x,-y)=-f_{ \pm}(x, y), \quad g_{ \pm}(x,-y)=g_{ \pm}(x, y),
$$

then system (41) is an isochronous focus if and only if the uncoupled systems $(\dot{x}, \dot{y})=$ $\left(f_{+}(x, y), g_{+}(x, y)\right)$ and $(\dot{x}, \dot{y})=\left(f_{-}(x, y), g_{-}(x, y)\right)$ are isochronous.

Remark. If condition (5) holds, then the phase portraits of the uncoupled systems are symmetric with respect to $y=0$. A system with this property is called a reversible center.

Proof. If the uncoupled systems are isochronous, then system (41) is an isochronous focus. Let us prove the converse. Without loss of generality we will assume that the origin is an attracting focus for the coupled system. Let $T_{+}(x)$ and $T_{-}(x)$ be the respective period maps of the uncoupled systems. For a given $x<0$ small enough, let $t_{+}(x)$ and $t_{-}(x)$ be the respective return times to the axis $y=0$ of the solutions of the uncoupled systems starting at $(x, 0)$. Because the focus is isochronous, there exists a constant $K>0$ such that

$$
t_{+}(x)+t_{-}(x)=K \quad \text { for all } x<0 .
$$

By the symmetry (5), we have that $t_{+}(x)=T_{+}(x) / 2$ and $t_{-}(x)=T_{-}(x) / 2$. Hence, $T_{+}(x)+T_{-}(x)=2 K$ for all $x<0$. As the fields are analytic and not degenerate at the origin, also $T_{+}$and $T_{-}$are analytic at 0 . Hence, $T_{+}(x)+T_{-}(x)=2 K$ for all $x$.

Given $x$, let us define $\sigma(x)$ as the first intersection point of the solution of the coupled system starting at $(x, 0)$ and the axis $y=0$. Then,

$$
\begin{aligned}
& t_{+}\left(\sigma^{2}(x)\right)+t_{-}(x)=\frac{1}{2}\left(T_{+}\left(\sigma^{2}(x)\right)+T_{-}(x)\right) \\
= & \frac{1}{2}\left(T_{+}(\sigma(x))+T_{-}(\sigma(x))\right)=K=t_{+}(x)+t_{-}(x) .
\end{aligned}
$$

Therefore, $t_{+}\left(\sigma^{2}(x)\right)=t_{+}(x)$ and $T_{+}(x)=T_{+}\left(\sigma^{2 n}(x)\right)$ for every $n \in \mathbb{N}$. Note that $\left\{\sigma^{2 n}(x)\right\}_{n}$ is a sequence converging to zero because the origin is an attracting focus. Since $T_{+}$is an analytic function at zero, which is constant on a sequence converging to zero, it is necessarily constant. Hence, $T_{-}(x)=2 K-T_{+}(x)$ is also constant.

Lemma 2.2. Let $f(x)=-x+\sum_{n \geq k} a_{n} x^{n}$ be an analytic involution with $k>1$ and $a_{k} \neq 0$. Then,

(1) $k$ is even,

(2) $a_{i}=0$ for every $i=k+1, k+3, \ldots, 2 k-3$,

(3) $a_{2 k-1}=\frac{-a_{k}^{2}}{2}$.

Proof. By definition, $(f \circ f)(x)=x$. Hence,

$$
\begin{aligned}
x= & (f \circ f)(x)=-f(x)+\sum_{n \leq k} a_{n} f(x)^{n} \\
= & x-a_{k} x^{k}-a_{k}(-x)^{k}-a_{k+1} x^{k+1}+a_{k+1}(-x)^{k+1}+\cdots \\
& \cdots-a_{2 k-1} x^{2 k-1}+a_{2 k-1}(-x)^{2 k-1}+k a_{k}^{2}(-x)^{k-1} x^{k}+O\left(x^{2 k}\right) .
\end{aligned}
$$

Identifying coefficients, we get $-a_{k}+(-1)^{k} a_{k}=0$. Hence, $k$ is even. Automatically, the odd coefficients up to $a_{2 k-3}$ are zero. Finally, the $(2 k-1)$-coefficient is $-2 a_{2 k-1}-k a_{k}^{2}=0$; therefore (3) holds. 
Theorem 2.1. Let $F(x)$ be an analytic function such that $F(x)=\sum_{n \geq k} a_{n} x^{n}$ with $k \geq 2$ and suppose that the planar system

$$
(\dot{x}, \dot{y})= \begin{cases}\left(-y, x+F^{\prime}(x)\right) & \text { if } y \geq 0 \\ \left(-y, x-F^{\prime}(x)\right) & \text { if } y<0\end{cases}
$$

is an isochronous focus. Then, $a_{n}=0$ for every $n>2$.

Proof. By Lemma2.1, both uncoupled systems $(\dot{x}, \dot{y})=\left(-y, x+F^{\prime}(x)\right)$ and $(\dot{x}, \dot{y})=$ $\left(-y, x-F^{\prime}(x)\right)$ must be isochronous. By theorem B of [6], there exist positive constants $k_{1}, k_{2}$ and involutions $\sigma_{1}, \sigma_{2}$ such that

$$
\begin{aligned}
& \frac{x^{2}}{2}+F(x)=k_{1}\left(x-\sigma_{1}(x)\right)^{2}, \\
& \frac{x^{2}}{2}-F(x)=k_{2}\left(x-\sigma_{2}(x)\right)^{2} .
\end{aligned}
$$

Let us write

$$
\begin{aligned}
& \sigma_{1}(x)=-x+\sum_{n \geq 2} b_{n} x^{n}=-x+l_{1}(x), \\
& \sigma_{2}(x)=-x+\sum_{n \geq 2} c_{n} x^{n}=-x+l_{2}(x) .
\end{aligned}
$$

Then, (7) reads as

$$
\begin{aligned}
& \frac{x^{2}}{2}+F(x)=k_{1}\left(2 x-l_{1}(x)\right)^{2}, \\
& \frac{x^{2}}{2}-F(x)=k_{2}\left(2 x-l_{2}(x)\right)^{2} .
\end{aligned}
$$

Therefore,

$$
4 k_{1}=1 / 2+a_{2}, \quad 4 k_{2}=1 / 2-a_{2} .
$$

Quadratic terms in (8) can be canceled. By adding the resulting equations, we obtain

$$
-4 x k_{1} l_{1}(x)-4 x k_{2} l_{2}(x)+k_{1} l_{1}^{2}(x)+k_{2} l_{2}^{2}(x)=0 .
$$

If $l_{1}$ and $l_{2}$ have a non-zero coefficient, then the first non-zero coefficients of both functions have the same index, say $k$. The coefficient corresponding to $x^{2 k}$ in (9) is

$$
-4 k_{1} b_{2 k-1}-4 k_{2} c_{2 k-1}+k_{1} b_{k}^{2}+k_{2} c_{k}^{2}=0 .
$$

By Lemma 2.2 it is known that $b_{2 k-1}<0$ and $c_{2 k-1}<0$; therefore, the previous term is negative. This is a contradiction. Hence, $l_{1}(x)=l_{2}(x)=0$ and $F(x)=$ $a_{2} x^{2}$.

\section{NON-TRIVIAL ISOCHRONOUS CENTERS}

The proof of Lemma 2.1 relies strongly on the fact that the origin is a focus. If assumption (iii) is removed, it could be possible to get a non-trivial isochronous center by matching two non-isochronous potentials.

Proposition 3.1. Given analytic potentials $V_{1}=a x^{2}+O\left(x^{2}\right)$ and $V_{2}=b x^{2}+$ $O\left(x^{2}\right)$, with $a>0$ and $b>0$, the coupled system

$$
(\dot{x}, \dot{y})= \begin{cases}\left(-y, V_{1}^{\prime}(x)\right) & \text { if } y \geq 0 \\ \left(-y, V_{2}^{\prime}(x)\right) & \text { if } y<0\end{cases}
$$

has a center at the origin if and only if there exists an analytic diffeomorphism at the origin $f$ such that $V_{2}=f\left(V_{1}\right)$. 
Proof. Let $V=k x^{2}+O\left(x^{2}\right)$ with $k>0$. Then the origin is a center for the associated potential system. Hence in a neighborhood of 0 we can implicitly define the map $\sigma_{V}(x)$ by

$$
V\left(\sigma_{V}(x)\right)=V(x)
$$

and $x \sigma_{V}(x) \leq 0$. Note that $\sigma_{V}$ is an involution. We claim that $\sigma_{V}$ is analytic at zero. To see this, consider the map

$$
g(x)=\operatorname{sgn}(x) \sqrt{V(x)}=x \sqrt{\frac{V(x)}{x^{2}}} .
$$

Since $k>0$, it follows that $g$ is analytic at 0 and $g^{\prime}(0)=\sqrt{k}$. Then $h(x)=$ $g^{-1}(-g(x))$ is also analytic at the origin. Furthermore, easy computations show that $V(h(x))=V(x)$, so $\sigma_{V}(x)=h(x)$ and the claim follows.

Clearly $\sigma_{V}^{\prime}(0)=-1$, and hence the map $p(x)=x-\sigma_{V}(x)$ is an analytic diffeomorphism at 0 . Then we can write

$$
V(x)=\tilde{V}\left(x-\sigma_{V}(x)\right),
$$

where $\tilde{V}=V \circ p^{-1}$ is also analytic at 0 . Since $V(x)=V\left(\sigma_{V}(x)\right)$ we get that

$$
\tilde{V}\left(x-\sigma_{V}(x)\right)=\tilde{V}\left(\sigma_{V}(x)-\sigma_{V}^{2}(x)\right)=\tilde{V}\left(-\left(x-\sigma_{V}(x)\right)\right) .
$$

Thus we have seen that $\tilde{V}$ is even, and therefore we obtain that

$$
V(x)=f_{V}\left(\left(x-\sigma_{V}(x)\right)^{2}\right) .
$$

Easy computations show that $f_{V}^{\prime}(0)=k / 4$. Hence $f_{V}$ is an analytic diffeomorphism at the origin.

Assume now that our system has a center at the origin. Then $\sigma_{V_{1}}=\sigma_{V_{2}}$, and we denote both maps by $\sigma$. From the above considerations we get that $V_{1}(x)=$ $f_{V_{1}}\left((x-\sigma(x))^{2}\right)$ and $V_{2}(x)=f_{V_{2}}\left((x-\sigma(x))^{2}\right)$ with $f_{V_{1}}, f_{V_{2}}$ analytic diffeomorphisms at the origin. Thus we obtain

$$
V_{2}(x)=f_{V_{2}}\left(f_{V_{1}}^{-1}\left(V_{1}(x)\right)\right) .
$$

Proposition 3.2. Let $F$ be an analytic function at 0 with $F(0)=F^{\prime}(0)=0$ and $\left|F^{\prime \prime}(0)\right|<1$. Then, the origin is a center for the planar system

$$
(\dot{x}, \dot{y})= \begin{cases}\left(-y, x+F^{\prime}(x)\right) & \text { if } y \geq 0 \\ \left(-y, x-F^{\prime}(x)\right) & \text { if } y<0\end{cases}
$$

if and only if $F$ is even.

Proof. Set $V_{1}(x)=x^{2} / 2+F(x)$ and $V_{2}(x)=x^{2} / 2-F(x)$. The conditions imposed on $F$ ensure that the corresponding planar systems associated to $V_{1}$ and $V_{2}$ have a center at the origin.

If $F$ is even, then $V_{1}$ and $V_{2}$ are both even functions. Therefore both potentials have the same associated involution, namely $\sigma(x)=-x$. So the coupled system (11) has a center at the origin.

Conversely, let us assume that system (11) has a center at the origin. By the last result there exists an analytic diffeomorphism $f$ such that

$$
\frac{x^{2}}{2}-F(x)=f\left(\frac{x^{2}}{2}+F(x)\right) \text {. }
$$


By contradiction, let us suppose that $\mathrm{F}$ is not even. Then, it has a first non-zero odd coefficient $a_{2 k+1}$. If we write $f(x)=\sum b_{n} x^{n}$, by setting equal the coefficients corresponding to $x^{2 k+1}$ in identity (12) we get a contradiction.

The next result states that a non-trivial isochronous center cannot be obtained by matching two isochronous potentials.

Lemma 3.1. There does not exist a non-trivial even function $F$ such that the potentials $V_{+}=\frac{x^{2}}{2}+F$ and $V_{-}=\frac{x^{2}}{2}-F$ are isochronous.

Proof. This follows from the fact that the only isochronous even potential is the linear one [6].

The following results state that it is not possible to generate a non-trivial isochronous center by matching two (non isochronous) centers generated by potentials $V_{+}=\frac{x^{2}}{2}+F$ and $V_{-}=\frac{x^{2}}{2}-F$.

Theorem 3.1. There does not exist an analytic non-linear function $F=\sum_{n \geq 3} a_{n} x^{n}$ such that the planar system (11) is an isochronous center.

Proof. Let us suppose that $F$ exists. By Proposition $3.2, F$ is even. For convenience let $F$ be re-indexed as $F(x)=\sum_{n \geq k} a_{n} x^{2 n}$, where $a_{k}$ is the first non-zero coefficient. We have that

$$
V_{+}(x)=\frac{x^{2}}{2}+F(x)=\frac{x^{2}}{2}+\sum_{n \geq k} a_{n} x^{2 n} .
$$

We will work in polar coordinates $(\theta, r)$. By hypothesis, we know that

$$
\int_{0}^{2 \pi} \frac{1}{\dot{\theta}(\theta, x)} d \theta=T(x)=\text { constant. }
$$

Our goal is to reach a contradiction by analyzing the power series expansion of $1 / \dot{\theta}$. Let us write

$$
r^{2}(\theta, x)=\sum_{n \geq 1} b_{n}(\theta) x^{2 n}
$$

Then,

$$
\frac{r^{2}(\theta, x)}{2}+\sum_{n \geq k} a_{n}\left[r^{2}(\theta, x)\right]^{n} \cos ^{2 n} \theta=\frac{x^{2}}{2}+\sum_{n \geq k} a_{n} x^{2 n} .
$$

By substituting (14) in (15) and equating the coefficients corresponding to the powers $2,4, \ldots, 2 k$, we get

$$
\begin{gathered}
b_{1}(\theta)=1, \\
b_{2}(\theta)=\cdots=b_{k-1}(\theta)=0, \\
b_{k}\left((\theta)=2 a_{k}\left(1-\cos ^{2 k} \theta\right) .\right.
\end{gathered}
$$

Therefore,

$$
\begin{aligned}
& r^{2}(\theta, x)=x^{2}+b_{k}(\theta) x^{2 k}+O(2 k+2), \\
& \dot{\theta}(\theta, x)=1+2 k a_{k} r^{2 k-2} \cos ^{2 k} \theta+O(2 k)=1+2 k a_{k} \cos ^{2 k} \theta x^{2 k-2} \\
& +\left(2 k(k-1) a_{k} b_{k} \cos ^{2 k} \theta+(4 k-2) a_{2 k-1} \cos ^{4 k-2} \theta\right) x^{4 k-4}+O(4 k-2) .
\end{aligned}
$$

It is easy to deduce that the coefficient of the power expansion of $1 / \dot{\theta}$ corresponding to $x^{4 k-4}$ is

$B^{+}(\theta)=4 k^{2} a_{k}^{2} \cos ^{4 k} \theta-4 k(k-1) a_{k}^{2}\left(1-\cos ^{2 k} \theta\right) \cos ^{2 k} \theta-(4 k-2) a_{2 k-1} \cos ^{4 k-2} \theta$. 
Hence, the coefficient corresponding to $x^{4 k-4}$ of $\int_{0}^{\pi} \frac{1}{\dot{\theta}(\theta, x)} d \theta$ is just $\int_{0}^{\pi} B^{+}(\theta) d \theta$.

By repeating the arguments with

$$
V_{-}(x)=\frac{x^{2}}{2}-F(x)=\frac{x^{2}}{2}-\sum_{n \geq k} a_{n} x^{2 n},
$$

we find that the coefficient corresponding to $x^{4 k-4}$ of $\frac{1}{\dot{\theta}(\theta, x)}$ is just

$B^{-}(\theta)=4 k^{2} a_{k}^{2} \cos ^{4 k} \theta-4 k(k-1) a_{k}^{2}\left(1-\cos ^{2 k} \theta\right) \cos ^{2 k} \theta+(4 k-2) a_{2 k-1} \cos ^{4 k-2} \theta$.

Now, the coefficient corresponding to $x^{4 k-4}$ of $\int_{0}^{2 \pi} \frac{1}{\dot{\theta}(\theta, x)} d \theta$ is

$$
\int_{0}^{\pi} B^{+}(\theta) d \theta+\int_{\pi}^{2 \pi} B^{-}(\theta) d \theta
$$

By (13), this coefficient must be zero. Further computations lead to the equality

$$
4 k^{2} \int_{0}^{\pi} \cos ^{4 k} \theta d \theta-4 k(k-1) \int_{0}^{\pi}\left(1-\cos ^{2 k} \theta\right) \cos ^{2 k} \theta d \theta=0 .
$$

Equivalently, we have that

$$
\frac{2 k-1}{k-1} \int_{0}^{\pi} \cos ^{4 k} \theta d \theta=\int_{0}^{\pi} \cos ^{2 k} \theta d \theta .
$$

It is now easy to prove that this equality does not hold for any $k$. For instance, we can integrate by parts to obtain

$$
\int_{0}^{\pi} \cos ^{4 k} \theta d \theta=\frac{(4 k-1)(4 k-3) \cdots(2 k+1)}{4 k(4 k-2) \cdots(2 k+2)} \int_{0}^{\pi} \cos ^{2 k} \theta d \theta .
$$

This, together with the previous equality, implies the identity

$$
(4 k-1)(4 k-3) \cdots(2 k+1)(2 k-1)=4 k(4 k-2) \cdots(2 k+2)(k-1) .
$$

For each $k$, its left-hand side is an odd integer while the right-hand side is an even integer. This gives the desired contradiction.

Proof of Theorem 1.1. It follows from Theorems 2.1 and 3.1.

Remark. Note that all arguments in the proof Theorem 1.1 use only the polynomial Taylor expansion (in fact the first non-zero coefficient). So Theorem 1.1 is true if $g$ is of class $C^{\infty}$ and $g^{(k)}(0) \neq 0$ for some $k>1$.

In what follows we will discuss a more general question. Let $F(x)=a x^{2}+$ $O\left(x^{3}\right)$ and $G(x)=b x^{2}+O\left(x^{3}\right), a, b>0$ be analytic functions defined on some neighborhood of 0 and consider the following system:

$$
(\dot{x}, \dot{y})= \begin{cases}\left(-y, F^{\prime}(x)\right) & \text { if } y \geq 0 \\ \left(-y, G^{\prime}(x)\right) & \text { if } y<0 .\end{cases}
$$

If the origin is a focus for the system (16), then it follows from Lemma 2.1 that it is an isochronous focus if and only if the associated uncoupled systems have an isochronous center at the origin. The following theorem shows that the situation is more complicated in the center case.

Theorem 3.2. Let $F(x)=a x^{2}+0\left(x^{4}\right), a>0$, be an analytic even function at 0 . Then for every $k>0$ there exists a unique analytic even function at the origin $G$, such that $G(x)=k x^{2}+O\left(x^{4}\right)$ and the system (16) has an isochronous center at the origin. 
Proof. Let $T(x)$ be the period function associated to the system

$$
(\dot{x}, \dot{y})=\left(-y, F^{\prime}(x)\right) .
$$

It is well known that $T$ is analytic at 0 and $T(0)=\sqrt{2 / a} \pi$. Moreover, $T(x)=$ $T(-x)$, so $T$ is even. Set $\tilde{T}(x)=T(x) / 4$. From the symmetries of the system (17), $\tilde{T}(x)$ can be viewed as the time needed by the solution with initial condition $(x, 0)$ to cross the $y$-axis. Set $k>0$ and

$$
\tilde{T}_{1}(x)=(\sqrt{2 / k}+\sqrt{2 / a}) \pi / 4-\tilde{T}(x) .
$$

Clearly $\tilde{T}_{1}(x)$ is even, analytic, and $\tilde{T}_{1}(0)>0$. From [1] it follows that there exists a unique function $G(x)$ such that the system

$$
(\dot{x}, \dot{y})=\left(-y, G^{\prime}(x)\right)
$$

has a center at the origin and, for all sufficiently small $x>0$, the time required for the solution of (18) with initial condition $(x, 0)$ to cross the $y$-axis is precisely $\tilde{T}_{1}(x)$. Moreover from [2] it follows that $G$ is analytic and even. It follows from the symmetries associated with the equation (18) that its period function $T_{1}$ satisfies the identity $T_{1}(x)=4 \tilde{T}_{1}(x)$. Then $T_{1}(0)=\sqrt{2 / k} \pi$ and so $G(x)=k x^{2}+O\left(x^{4}\right)$. Since $F$ and $G$ are even it follows that system (16) has a center at the origin and clearly its period function $T_{2}$ satisfies the equality $T_{2}(x)=T(x) / 2+T_{1}(x) / 2=$ $(\sqrt{2 / k}+\sqrt{2 / a}) \pi / 2$.

We suspect that the above theorem also holds in the non-even case. Unfortunately our attempts to prove it have failed.

\section{ACKNOWLEDGMENT}

The second author wishes to thank Rafael Ortega for suggesting the study of reference [3].

\section{REFERENCES}

[1] B. Alfawicka, Inverse problems connected with periods of oscillations described $\ddot{x}+g(x)=0$. Ann. Polon. Math. 44 (1984), no. 3, 297-308. MR0817804 (87d:34015)

[2] B. Alfawicka, Inverse problem connected with half-period function analytic at the origin. Bull. Polish Acad. Sci. Math. 32 (1984), no. 5-6, 267-274. MR0785984 (86e:34028)

[3] J. Andrade, Les frottements et l'isochronisme, C. R. Acad. Sci. Paris, (1920), 664-665.

[4] C. Chicone, The monotonicity of the period function for planar Hamiltonian vector fields. $J$. Differential Equations 69 (1987), no. 3, 310-321. MR0903390 (88i:58050)

[5] A. Cima, A. Gasull and F. Mañosas, Period function for a class of Hamiltonian systems. J. Differential Equations 168 (2000), no. 1, 180-199. MR1801350 (2001m:34068)

[6] A. Cima, F. Mañosas and J. Villadelprat, Isochronism for several classes of Hamiltonian systems, J. Differential Equations, 157 (1999), 373-413. MR1713265(2000h:34073)

[7] B. Coll, A. Gasull and R. Prohens, Center-focus and isochronous center problems for discontinuous differential equations. Discrete Contin. Dynam. Systems 6 (2000), no. 3, 609-624. MR 1757390 (2001f:34053)

[8] K. Deimling and P. Szilágyi, Periodic solutions of dry friction problems, Z. angew. Math. Phys. 45 (1994), 53-60. MR 1259526 (94m:34099)

[9] L. Gavrilov, Isochronism of plane polynomial Hamiltonian systems. Nonlinearity 10 (1997) 433-448. MF,1438261 (98b:58143)

[10] R.C. Hibbeler, Mechanics for engineers, MacMillan Publishing Company, 1985. 
[11] M. Urabe, The potential force yielding a periodic motion whose period is an arbitrary continuous function of the amplitude of the velocity. Arch. Rational Mech. Anal. 11 (1962), 27-33 MR.0141834(25:5231)

[12] M. Urabe, Potential forces which yield periodic motions of a fixed period. J. Math. Mech. 10 (1961), 569-578. MR0123060 (23:A391)

Departament de Matematiques, Universitat Autonoma de Barcelona, 08193 Bellaterra, Barcelona, Spain

E-mail address: Francesc.Manosas@uab.es

Departamento de Matemática Aplicada, Universidad de Granada, 18071 Granada, SPAIN

E-mail address: ptorres@ugr.es 